\title{
Association between Plasma Myeloperoxidase and Free 3-Nitrotyrosine Levels in Patients with Coronary Artery Disease
}

\author{
Morteza Pourfarzam ${ }^{1}$, Ahmad Movahedian², Nizal Sarrafzadegan ${ }^{1}$, Gholam Basati ${ }^{3,4 *}$ \\ Saed Ziaaldin Samsamshariat ${ }^{2}$ \\ ${ }^{1}$ Cardiovascular Research Center, Isfahan Cardiovascular Research Institute, Isfahan University of Medical Sciences, Isfahan, Iran; \\ ${ }^{2}$ Pharmaceutical Sciences Research Centre, Department of Clinical Biochemistry, School of Pharmacy and Pharmaceutical Sciences, \\ Isfahan University of Medical Sciences, Isfahan, Iran; ${ }^{3}$ Department of Clinical Biochemistry, Faculty of Medicine, Ilam University of \\ Medical Sciences, Ilam, Iran; ${ }^{4}$ Clinical Microbiology Research Center, Ilam University of Medical Sciences, Ilam, Iran. \\ Email:"gholam_basati@yahoo.com, ${ }^{*}$ basati-gh@medilam.ac.ir
}

Received December $3^{\text {rd }}, 2012$; revised February $5^{\text {th }}, 2013$; accepted March $11^{\text {th }}, 2013$

\begin{abstract}
Objective: Myeloperoxidase (MPO) is an inflammatory enzyme that is mainly released by activated neutrophils and monocytes. 3-nitrotyrosine (NT) is a stable inflammatory end product of MPO that is produced through nitrosylation of free and protein-bound tyrosines. Determination of the exact levels of free NT is technically a challenging matter. Also, there is limited information about the relationship between MPO and free NT levels and elevation of them in the plasma of patients with coronary artery disease (CAD). Therefore, we sought to determine the exact level of plasma free NT with a simple and exquisite technique in CAD patients. Methods: This study included 50 stable angina, 50 unstable angina patients, and 50 control subjects. Plasma MPO concentration was measured with an immunoassay method. Plasma free NT level was determined by a modified HPLC-fluorescence method. Lipid profile, high sensitivity C-reactive protein (hsCRP) and other clinical risk factors of patients were also assigned. Results: Plasma level of free NT was efficiently measured by the HPLC-fluorescence method. Plasma levels of MPO and NT were significantly higher in patients with stable and unstable CAD than in control subjects $(\mathrm{P}<0.001)$. There was a significant correlation between the two substances in CAD patients $(\mathrm{P}<0.001)$. Conclusions: We determined plasma free NT levels with a sensitive HPLC-fluorescence method with some modifications in a clinical scale. Plasma levels of MPO and NT were profoundly elevated in CAD patients. The significant relationships of the two substances and elevation of them may have useful clinical implication in patients with stable and unstable CAD.
\end{abstract}

Keywords: Myeloperoxidase; 3-Nitrotyrosine; Stable; Unstable CAD

\section{Introduction}

Myeloperoxidase (MPO) is an inflammatory enzyme that is mainly produced by neutrophils and monocytes. The enzyme plays a pivotal role in atherogenesis through production of many oxidized metabolites [1-4].

It is currently apparent that 3-nitrotyrosine (NT) as a molecular fingerprint for MPO-catalyzed oxidation may have an important implication in cardiovascular diseases [5]. Elevation of NT in the atheromatous plaques as demonstrated by immunohistochemistry depicted direct involvement of NT in atherosclerosis [6,7].

MPO nitrosylates the tyrosine residues of apo A-I component of high density lipoprotein (HDL) and thereby interferes with cholesterol efflux from macrophages

"Corresponding author.
[8]. Accordingly, the amount of plasma nitrosylated HDL in patients with coronary artery disease (CAD) is nearly two fold of those in control subjects [6]. Also, MPO produces reactive nitrogen species that convert low density lipoprotein (LDL) into an atherogenic form. This modified LDL is endocytosed by macrophages, leading to foam cell transformation [9]. The colocalization of both MPO and nitrotyrosine in the subendothelial space of arteries [10] further stressed the role of MPO and NT in inflammatory diseases, such as CAD.

Also, the association of plasma protein-bound NT levels with the presence of CAD has reported by one study [11].

Plasma free NT is the result of nitroslyated proteins turnover and may reflect the amount of plasma nitroslyated protein therefore, quantization of free NT is an appropriate strategy to assess nitroxidative stress [12]. 
Measurement of plasma free NT in relation to MPO in CAD patients may further delineated importance of the biomarkers in clinical scale. Therefore, in the present study, we decided to evaluate the relationship of MPO and free NT in stable and unstable CAD patients as compared with control subjects.

\section{Materials and Methods}

\subsection{Patients}

Subjects included in this study were consecutively recruited in Isfahan Cardiovascular Research Center in Iran in a double blinded fashion. In brief, 50 patients with stable angina, 50 patients with unstable angina, and 50 control subjects were assigned for the study based on angiography. In all CAD patients coronary artery stenosis was $\geq 50 \%$ in at least one of the three main coronary arteries. Stable CAD patients had a chest pain that usually experienced on exertion and relived on rest. Unstable CAD patients had an unexpected chest pain that prolonged for about thirty minutes and did not respond to usual medication. Control subjects had no any history of cardiac diseases and a majority of them had a coronary artery stenosis less than $10 \%$. Written informed consent for all subjects was prepared and the approval of the study was confirmed by the ethical committee of the Center. Patients with myocardial infarction or cardiovascular events, surgery, cancer, chronic or acute inflammatory diseases were not recruited in the study.

Information about traditional clinical risk factors was obtained by using of a standardized questionnaire after admission of patients. Hypertension was assigned based on systolic blood pressure $\geq 140 \mathrm{~mm} / \mathrm{Hg}$ and diastolic blood pressure $\geq 90 \mathrm{~mm} / \mathrm{Hg}$, or use of antihypertensive medication. Diabetes mellitus was determined as a fasting serum glucose $\geq 126 \mathrm{mg} / \mathrm{dl}$ and hemoglobin $\mathrm{A}_{1 \mathrm{c}} \geq$ $6.5 \%$, or use of hypoglycemic medications. Family history of CAD was revealed as CAD events in first-degree relatives at $<55$ years of age in men or $<65$ years in women. Smoking was also revealed as the use of any form of tobacco and cigarette in any quantity. The consumption of cardiac drugs in the subjects was also explored.

\subsection{MPO Assay}

Fasting EDTA-plasma sample was prepared from all subjects, aliquoted and maintained at $-80^{\circ} \mathrm{C}$ until analyzed. Plasma MPO amounts were measured with an ELISA assay kit (Immunology Consultants Laboratory, Inc., Newberg, OR, USA). The intra-assay and interassay coefficient of variation for the kit was $2.8 \%$ and $6.5 \%$, respectively. Also the detection limit and recovery of the assay was $0.130 \mathrm{ng} / \mathrm{mL}$ and $86.7 \%$, respectively.
Plasma level of high sensitivity $\mathrm{C}$ reactive protein (hsCRP) was determined with a latex-enhanced immunoturbidometric assay (Roche Diagnostics, Mannheim, Germany). Plasma lipid profile (total cholesterol, triglycerides, LDL-cholesterol and high-density lipoprotein cholesterol) was determined using standard enzymatic kits (Parsazmun, Karaj, Iran).

\subsection{Free NT Assay}

In the study, we assayed plasma free NT based on Kamisaki et al. [13] method with some modifications as follow:

The chromatography equipment was a Shimadzu LC system, model 10Avp (Tokyo, Japan) consisting of two pump (LC-10ADvp), a fluorescence detector (RF10AXL), a system controller (SCL-10Avp) and a personal computer with CLASS-VP software (Shimadzu, Kyoto, Japan). A Nova-Pak C18 column $(3.9 \times 150 \mathrm{~mm}$; $4 \mu \mathrm{m}$ particle size; $60 \AA$ pore size) accompanied also with a Nova-Pak C18 guard column $(2 \times 20 \mathrm{~mm} ; 4 \mu \mathrm{m}$ particle size; $80 \AA$ pore size) were used.

For each subjects, $60 \mu \mathrm{L}$ EDTA-plasma sample was mixed with $140 \mu \mathrm{L}$ of cold ethanol and $20 \mu \mathrm{L} \alpha$-Lmethyl tyrosine (MT) $(1.815 \mu \mathrm{M})$, as an internal standard. This mixture was vortexed toughly and kept on ice for 20 minutes. After centrifugation at $18,000 \mathrm{rpm}$ for $15 \mathrm{~min}$ at $4^{\circ} \mathrm{C}$ the resulting supernatant was kept at $-20^{\circ} \mathrm{C}$ until the chromatographic analysis.

For derivatisation, $100 \mu \mathrm{L}$ of the stored supernatant (after thawing at room temperature) were derivatized with $20 \mu \mathrm{L}$ of $0.45 \mathrm{M}$ sodium borate buffer ( $\mathrm{pH} 9.5$ ) and 30 $\mu \mathrm{L}$ of 4-Fluoro-7-nitrobenzo-2-oxa-1,3-diazole (NBD-F) (10 $\mathrm{mM}$ in acetonitrile), incubated at $60^{\circ} \mathrm{C}$ for $2 \mathrm{~min}$. The reaction was stopped by addition of $15 \mu \mathrm{L}$ of $0.5 \mathrm{M} \mathrm{HC}$.

The chromatographic elution was accomplished with injection of $25 \mu \mathrm{L}$ of the derivitized supernatant at room temperature with a mobile phase consisting of sodium phosphate buffer $(0.1 \mathrm{M}, \mathrm{pH}$ 7.2) and methanol (52.5: $47.5 \mathrm{v} / \mathrm{v}$ ) at an isocratic flow rate of $1.0 \mathrm{ml} / \mathrm{min}$. The fluorescence detector was set at excitation and emission wavelengths of 470 and $540 \mathrm{~nm}$, respectively. The typical retention times of NT and MT were 18 min and 32 min, respectively. After every 10 sample analyses, the column was first rinsed with water-methanol $(50: 50 \mathrm{v} / \mathrm{v})$ for $20 \mathrm{~min}$ and then with water-acetonitrile $(15: 85 \mathrm{v} / \mathrm{v})$ for $30 \mathrm{~min}$ at the above flow rate.

A calibration curve (consisting of $0,1,2,5,10,15,15$, 25 and $50 \mathrm{nM}$ of spiked authentic NT) was prepared by plotting the peak area of total NT over MT (minus that of the blank plasma) in each calibrator against the concentration of spiked NT. This calibration curve was applied for the calculation of NT in all samples.

The recovery of this method was $105 \%$. The limit of 
quantification (LOQ) based on the signal to noise ratio of 10:1 was calculated at $0.6 \mathrm{nM}$. The limit of detection (LOD) based on the signal to noise ratio of 3:1 was about $0.2 \mathrm{nM}$. Also, the intra-assay and inter-assay coefficient of variation for the method were $4.2 \%$ and $7.3 \%$, respectively.

\section{Statistical Analyses}

All of the statistical evaluations were carried out using SPSS statistical software version 16.0 (SPSS Inc., Chicago, IL, USA). Correlations between the plasma concentrations of MPO and NT were assessed by Pearson's coefficient of correlation (r). All data were reported either as mean \pm standard deviation or as counts and percentages. A P-value $<0.05$ was considered to be statistically significant.

\section{Results}

The clinical characteristics of the patients are presented in Table 1.

Patients and control subjects were age and sex matched. A higher percentage of patients had cardiovascular risk factors.

As depicted in Figure 1, the correlation between plas- ma MPO and free NT levels in unstable CAD $(r=0.612$, $\mathrm{P}=0.01)$ and stable $\operatorname{CAD}(\mathrm{r}=0.549, \mathrm{P}=0.01)$ patients is significant. The correlation is weak in control subjects $(\mathrm{r}$ $=0.29, \mathrm{P}=0.05$ ).

Typical chromatograms of a sample with and without authentic NT and MT are represented in Figure 2.

The peaks of NT and MT were identified based on the retention time of the authentic NT and MT and the spiking of them in plasma.

\section{Discussion}

In the present study, we clearly depicted that there is a significant association between plasma MPO and free NT levels in patients with stable and unstable CAD.

Elevation of NT in patients with oxidative stress condition such as diabetes and smoking has been demonstrated [7]. There are limited clinical investigations about the elevation of NT and its relation to MPO in cardiovascular disease. However, it is currently appeared that NT as an oxidative by-product of MPO is detectable in plasma and may be used as diagnostic and prognostic biomarkers of cardiovascular disease [11,14]. The elevations of MPO and NT and the correlation of them in stable and unstable CAD patients in the present study are in

Table 1. Clinical characteristic of patients.

\begin{tabular}{|c|c|c|c|c|}
\hline Variable & Control subjects $(\mathrm{n}=50)$ & Stable CAD patients $(\mathrm{n}=50)$ & Unstable CAD patients $(\mathrm{n}=50)$ & $P$ value \\
\hline Age (year) & $58.9 \pm 10.3$ & $61.2 \pm 11.23$ & $59.9 \pm 10.45$ & 0.51 \\
\hline Men & $35(70 \%)$ & $28(56 \%)$ & $32(64 \%)$ & 0.35 \\
\hline $\operatorname{BMI}\left(\mathrm{kg} / \mathrm{m}^{2}\right)$ & $26.9 \pm 0.6$ & $27.8 \pm 1.5$ & $26.89 \pm 1.3$ & 0.387 \\
\hline Cholesterol (mg/dL) & $178.3 \pm 47.0$ & $192.9 \pm 36.3$ & $199.5 \pm 55.8$ & 0.073 \\
\hline Triglycerid (mg/dL) & $147.4 \pm 36.3$ & $159.3 \pm 80.5$ & $161.9 \pm 95.6$ & 0.073 \\
\hline $\mathrm{LDL}(\mathrm{mg} / \mathrm{dL})$ & $96.8 \pm 27.5$ & $109.4 \pm 23.8$ & $113.5 \pm 31.5$ & $0.009^{\mathrm{a}}$ \\
\hline HDL (mg/dL) & $46.1 \pm 9.5$ & $44.7 \pm 9.6$ & $41.8 \pm 11$ & 0.094 \\
\hline hsCRP (mg/L) & $1.9 \pm 1.3$ & $3.2 \pm 1.4$ & $4.0 \pm 1.7$ & 0.03 \\
\hline MPO (ng/mL) & $23.0 \pm 3.6$ & $34.5 \pm 6.8$ & $71.2 \pm 19.6$ & 0.0001 \\
\hline NT (nM) & $4.4 \pm 1.8$ & $12.8 \pm 3.9$ & $14.8 \pm 4.8$ & 0.015 \\
\hline Hypertension & $3(6)$ & $20(40)$ & $20(40)$ & $0.0001^{\mathrm{b}}$ \\
\hline Diabetes mellitus & $1(2)$ & $12(24)$ & $20(40)$ & $0.01^{\mathrm{b}}$ \\
\hline Family history of CAD & $10(20)$ & $20(40)$ & $30(60)$ & $0.0001^{\mathrm{a}}$ \\
\hline Smoking & $2(4)$ & $8(16)$ & $15(30)$ & $0.01^{\mathrm{b}}$ \\
\hline Lipid-lowering agents & $2(4)$ & $18(36)$ & $28(56)$ & 0.022 \\
\hline Aspirin & $0(0)$ & $28(56)$ & $32(64)$ & $0.0001^{\mathrm{b}}$ \\
\hline
\end{tabular}

Data are expressed as mean \pm SD or number (\%), BMI: body mass index, LDL: low density lipoprotein, HDL: high density lipoprotein, hsCRP: high sensitive


vs Stable and Unstable CAD patients. 




(a)



(b)



(c)

Figure 1. Bivariate correlations between plasma myeloperoxidase (MPO) and free 3-nitrotyrosine (NT) levels in unstable CAD and stable CAD patients (panel (a) and (b), respectively) and control subjects (panel (c)). line with the above notion. Especially, we found that in acute condition of CAD such as unstable CAD, the two biomarkers were elevated more significantly. In this regard, our finding corroborated the dependence of nitrosative stress with the severity of CAD, because some antiinflammatory drugs such as statins have been able to decrease the CAD severity and NT levels [14]. Also, elevation of MPO in CAD patients with non obstructive culprit plaques further reflects the importance of oxidative damages and coronary artery sequelae [15]. The mechanisms of NT damage is not clearly defined but it has been proposed that nitrosylated LDL are more selectively recognized by the scavenger receptor CD36 [16] that lead to fatty streak formation and atherosclerotic lesion development [17]. Also, nitrosylated LDL induces the non differentiated human monocytes to release TNF- $\alpha$ and thereby potentiate inflammatory process in vivo [18]. Furthermore, nitrotyrosine modification of apolipoprotein A-I leads to the dysfunction of HDL in subjects with cardiovascular disease $[8,19]$. In fact, the content of NT residues in HDL is about $7-70$ fold of the content of NT residues in whole plasma protein in patients with CAD $[6,19]$.

Although plasma free NT have been measured by many methods, but each methods have its own advantages and drawbacks [20].

Precolumn derivatization of NT with NBD-F for determination of it in plasma, has been reported by some studies $[13,21,22]$. In the present study, precolumn derivatization of NT with NBD-F has been applied for the measurements of plasma free NT in a relatively large number of patients as compared to the former studies. The amount of NT in control subjects was in good agreement with the results of Zhang et al. [21] that also have been obtained by HPLC-fluorescence. Also, results of the present study are comparable with those obtained by other techniques [23]. The values that have been reported for the concentration of free NT in plasma vary considerably [24]. One of the problems with the studies about free NT level may be that the number of subjects under study is insufficient to conclude a decisive result about the amount of free NT in normal plasma. Also, it should be considered that some elevation of NT in healthy asymptomatic individual without inflammatory disease are also plausible [25]. In the present study, our overall method is based on the method of Kamisaki et al. [13], however for the better separation of NT, we exerted some modifications. By evaluation of different ratios of the mobile phase methanol: phosphate buffer, we found that the best ratio for the exact and efficient separation of NT and MT is 48:52 v/v of methanol:phosphate buffer. The derivatization condition in the present study is, in part, based on the procedure of Guo et al. [22], however 


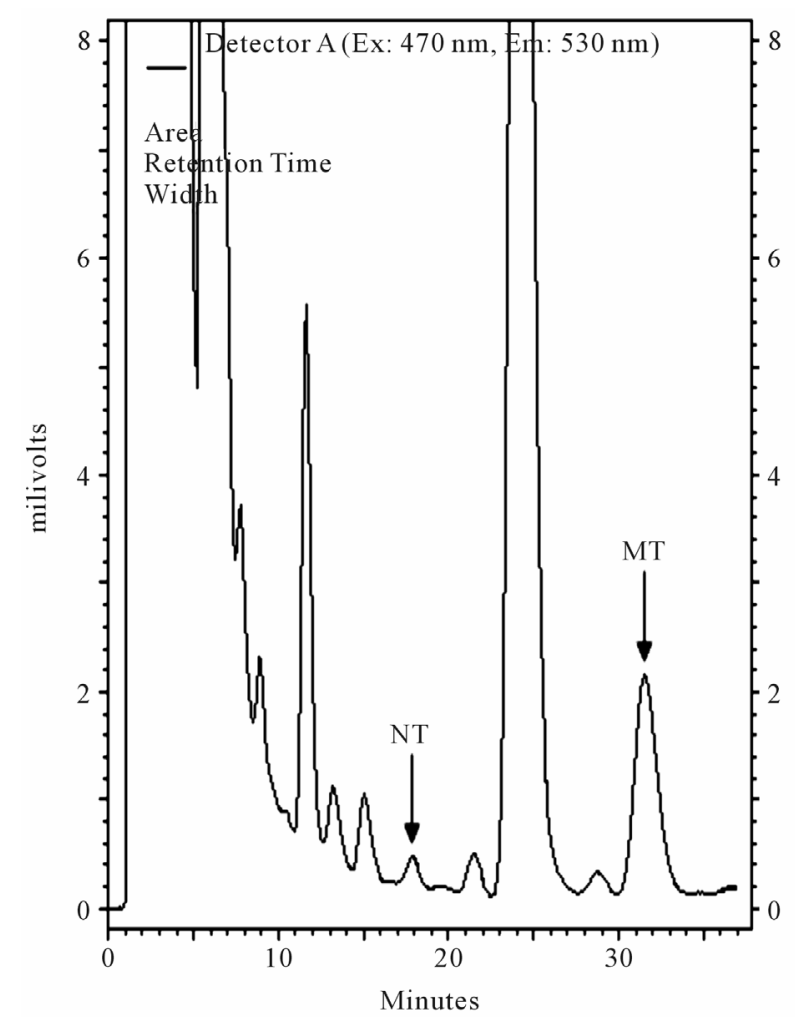

(a)

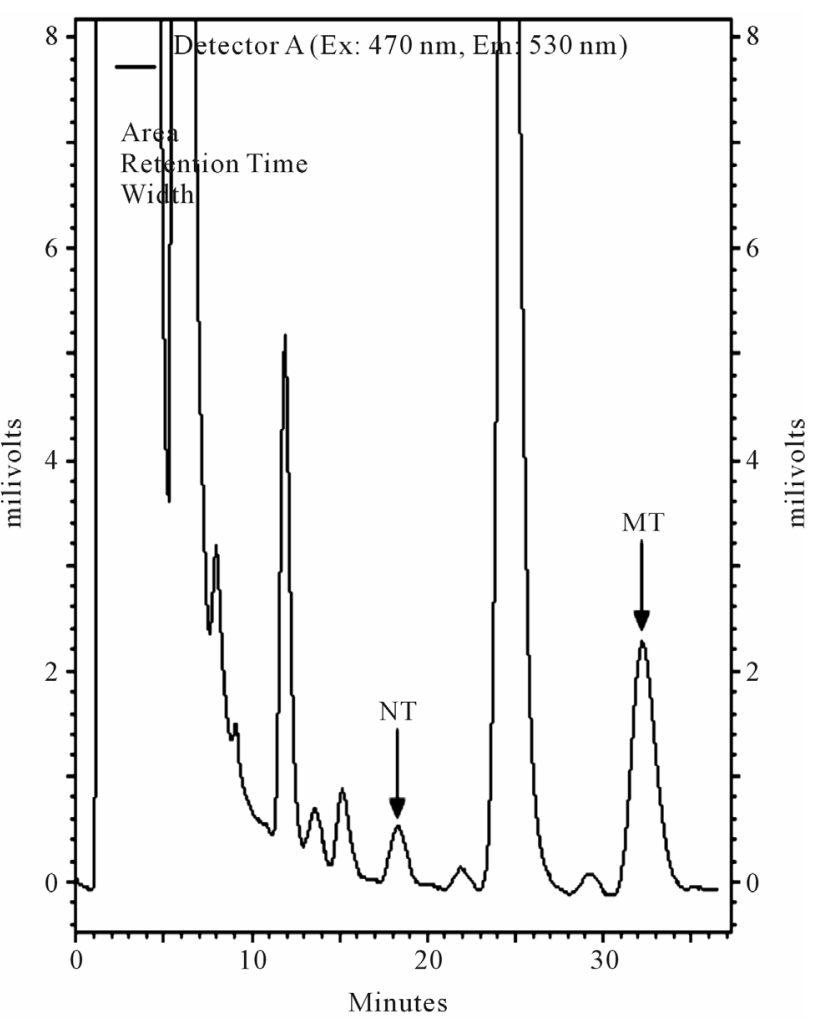

(b)

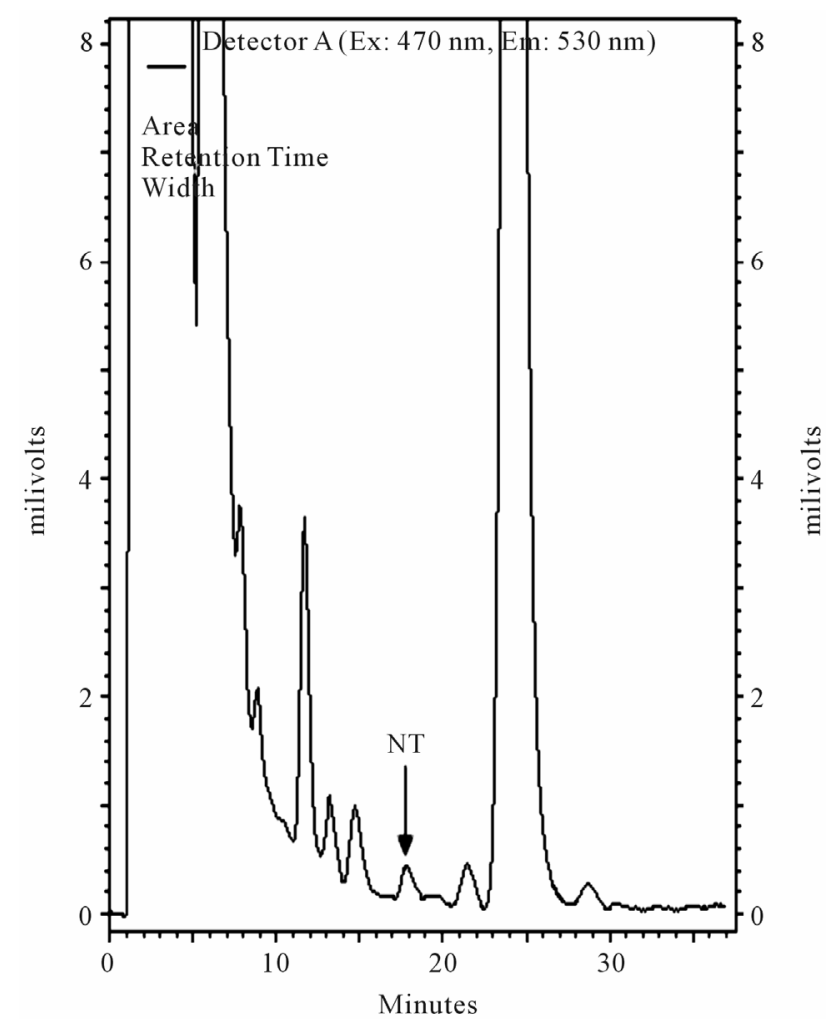

(c)

Figure 2. Typical chromatograms of a plasma sample spiked with 3-nitrotyrosine (NT) and $\alpha$-methyl tyrosine (MT); (a) Only $100 \mathrm{nM}$ of MT was spiked; (b) $100 \mathrm{nM}$ of MT and $10 \mathrm{nM}$ of NT were spiked; (c) Neither was spiked. 
we directly applied the supernatant for derivatization and had not any vaporization under nitrogen gas and SepPack extraction stages. Altogether, this modified procedure is an improved, simple and highly sensitive HPLC method that measures free NT in plasma for clinical purposes.

In summary, according to the results of the study there was a significant correlation between plasma MPO and free NT levels in CAD patients and the correlation was higher in acute condition such as unstable CAD.

\section{Acknowledgements}

This study was funded by grant number 388417 for $\mathrm{PhD}$ thesis from Isfahan University of Medical Sciences, Isfahan, Iran.

\section{REFERENCES}

[1] C. C. Winterbourn and A. J. Kettle, "Biomarkers of Myeloperoxidase-derived Hypochlorous Acid," Free Radical Biology and Medicine, Vol. 29, No. 4, 2000, pp. 403-409. doi:10.1016/S0891-5849(00)00204-5

[2] A. C. Carr, M. R. McCall and B. Frei, "Oxidation of LDL by Myeloperoxidase and Reactive Nitrogen Species: Reaction Pathways and Antioxidant Protection," Arteriosclerosis, Thrombosis, and Vascular Biology, Vol. 20, No. 7, 2000, pp. 1716-1723. doi:10.1161/01.ATV.20.7.1716

[3] S. J. Klebanoff, "Myeloperoxidase," Proceedings of the Association of American Physicians, Vo. 111, No. 5, 1999, pp. 383-389.

[4] Z. Prokopowicz, J. Marcinkiewicz, D. R. Katz and B. M. Chain, "Neutrophil Myeloperoxidase: Soldier and Statesman," Archivum Immunologiae et Therapiae Experimentalis, Vol. 60, No. 1, 2012, pp. 43-54. doi:10.1007/s00005-011-0156-8

[5] I. Mohiuddin, H. Chai, P. H. Lin, A. B. Lumsden, Q. Yao and C. Chen, "Nitrotyrosine and Chlorotyrosine: Clinical Significance and Biological Functions in the Vascular System," Journal of Surgical Research, Vol. 133, No. 2, 2006, pp. 143-149. doi:10.1016/j.jss.2005.10.008

[6] S. Pennathur, C. Bergt, B. Shao, J. Byun, S. Y. Kassim, P. Singh, P. S. Green, T. O. McDonald, J. Brunzell, A. Chait, J. F. Oram, K. O'brien, R. L. Geary and J. W. Heinecke. "Human Atherosclerotic Intima and Blood of Patients with Established Coronary Artery Disease Contain High Density Lipoprotein Damaged by Reactive Nitrogen Species," Journal of Biological Chemistry, Vol. 279, No. 41, 2004, pp. 42977-42983. doi:10.1074/jbc.M406762200

[7] M. H. Zou, R. A. Cohen and V. Ullrich, "Peroxynitrite and Vascular Endothelial Dysfunction in Diabetes Mellitus," Endothelium, Vol. 11, No. 2, 2004, pp. 89-97. doi:10.1080/10623320490482619

[8] L. Zheng, M. Settle, G. Brubaker, D. Schmitt, S. L. Hazen, J. D. Smith and M. Kinter, "Localization of Nitration and Chlorination Sites on Apolipoprotein AI Catalyzed by Myeloperoxidase in Human Atheroma and As- sociated Oxidative Impairment in ABCA1-Dependent Cholesterol Efflux from Macrophages," Journal of Biological Chemistry, Vol. 280, No. 1, 2005, pp. 38-47.

[9] E. A. Podrez, D. Schmitt, H. F. Hoff and S. L. Hazen, "Myeloperoxidase-Generated Reactive Nitrogen Species Convert LDL into an Atherogenic Form in Vitro," Journal of Clinical Investigation, Vo. 103, No. 11, 1999, pp. 1547-1560. doi:10.1172/JCI5549

[10] S. Baldus, J. P. Eiserich, M. L. Brennan, R. M. Jackson, C. B. Alexander and B. A. Freeman, "Spatial Mapping of Pulmonary and Vascular Nitrotyrosine Reveals the Pivotal Role of Myeloperoxidase as a Catalyst for Tyrosine Nitration in Inflammatory Diseases," Free Radical Biology and Medicine, Vol. 33, No. 7, 2002, pp. 1010-1019. doi:10.1016/S0891-5849(02)00993-0

[11] M. H. Shishehbor, R. J. Aviles, M. L. Brennan, X. Fu, M. Goormastic and G. L. Pearce, "Association of Nitrotyrosine Levels with Cardiovascular Disease and Modulation by Statin Therapy," Journal of the American Medical Association, Vol. 289, No. 13, 2003, pp. 1675-1680. doi:10.1001/jama.289.13.1675

[12] G. Peluffo and R. Radi, "Biochemistry of Protein Tyrosine Nitration in Cardiovascular Pathology," Cardiovascular Research, Vol. 75, No. 2, 2007, pp. 291-302. doi:10.1016/j.cardiores.2007.04.024

[13] Y. Kamisaki, K. Wada, K. Nakamoto, Y. Kishimoto, M. Kitano and T. Itoh, "Sensitive Determination of Nitrotyrosine in Human Plasma by Isocratic High-Performance Liquid Chromatography," Journal of Chromatography B: Biomedical Sciences and Applications, Vol. 685, No. 2, 1996, pp. 343-347. doi:10.1016/S0378-4347(96)00202-2

[14] M. H. Shishehbor, M. L. Brennan, R. J. Aviles, X. Fu, M. S. Penn, D. L. Sprecher and S. L. Hazen, "Statins Promote Potent Systemic Antioxidant Effects through Specific Inflammatory Pathways," Circulation, Vol. 108, No. 4, 2003, pp. 426-431. doi:10.1161/01.CIR.0000080895.05158.8B

[15] Z. H. Hou, B. Lu, Y. Gao, H. L. Cao, F. F. Yu, N. Jing, et al., "Matrix Metalloproteinase-9 and Myeloperoxidase Levels in Patients with Nonobstructive Coronary Artery Disease Detected by Coronary Computed Tomographic Angiography," Academic Radiology, Vol. 20, No. 1, 2012, pp. 25-31.

[16] E. A. Podrez, M. Febbraio, N. Sheibani, D. Schmitt, R. L. Silverstein, D. P. Hajjar, P. A. Cohen, W. A. Frazier, H. F. Hoff and S. L. Hazen, "Macrophage Scavenger Receptor CD36 Is the Major Receptor for LDL Modified by Monocyte-generated Reactive Nitrogen Species," Journal of Clinical Investigation, Vol. 105, No. 8, 2000, pp. 10951108. doi:10.1172/JCI8574

[17] M. Febbraio, E. A. Podrez, J. D. Smith, D. P. Hajjar, S. L. Hazen and H. F. Hoff, "Targeted Disruption of the Class B Scavenger Receptor CD36 Protects against Atherosclerotic Lesion Development in Mice," Journal of Clinical Investigation, Vol. 105, No. 8, 2000, pp. 1049-1056. doi:10.1172/JCI9259

[18] C. D. W. Smythe, V. O. Skinner, K. R. Bruckdorfer, D. O. Haskard and R. C. Landis, "The State of Macrophage 
Differentiation Determines the TNF [Alpha] Response to Nitrated Lipoprotein Uptake," Atherosclerosis, Vol. 170, No. 2, 2003, pp. 213-221. doi:10.1016/S0021-9150(03)00285-5

[19] L. Zheng, B. Nukuna, M. L. Brennan, M. Sun, M. Goormastic, M. Settle, D. Schmitt, X. Fu, L. Thomson, P. L. Fox, H. Ischiropoulos, J. D. Smith, M. Kinter and S. L. Hazen, "Apolipoprotein AI Is a Selective Target for Myeloperoxidase-Catalyzed Oxidation and Functional Impairment in Subjects with Cardiovascular Disease," Journal of Clinical Investigation, Vol. 114, No. 4, 2004, pp. 529-541.

[20] D. Tsikas, E. Schwedhelm and J. C. Frolich, "Methodological Considerations on the Detection of 3-Nitrotyrosine in the Cardiovascular System," Circulation Research, Vol. 90, No. 6, 2002, p. e70. doi:10.1161/01.RES.0000014802.05780.AE

[21] W. Z. Zhang, C. Lang and D. M. Kaye, "Determination of Plasma Free 3-Nitrotyrosine and Tyrosine by Reversedphase Liquid Chromatography with 4-fluoro-7-Nitrobenzofurazan Derivatization," Biomedical Chromatography, Vol. 21, No. 3, 2007, pp. 273-278.

doi:10.1002/bmc. 750
[22] S. Guo, L. Shao, J. Pei, L. Rong, X. Wang and D. Chi, "Determination of Free 3-Nitrotyrosine in Human Plasma Using HPLC Method with Fluorescence Detection," Chemia Analityczna, Vol. 54, No. 4, 2009, pp. 691-703.

[23] A. S. Pannala, A. R. Mani, J. P. E. Spencer, V. Skinner, K. R. Bruckdorfer, K. P. Moore and C. A. Rice-Evans, "The Effect of Dietary Nitrate on Salivary, Plasma, and Urinary Nitrate Metabolism in Humans," Free Radical Biology and Medicine, Vol. 34, No. 5, 2003, pp. 576-584. doi:10.1016/S0891-5849(02)01353-9

[24] H. Ryberg and K. Caidahl, "Chromatographic and Mass Spectrometric Methods for Quantitative Determination of 3-Nitrotyrosine in Biological Samples and Their Application to Human Samples," Journal of Chromatography B, Vol. 851, No. 1-2, 2007, pp. 160-171. doi:10.1016/j.jchromb.2007.02.001

[25] L. L. Wu and J. T. Wu, "Serum 3-Nitrotyrosine May Be Elevated with the Exposure to Acute Inflammatory Risk Factors Such as Unhealthy Diet, Pollutant, Drug and Psychosocial Stress," Journal of Biomedical Laboratory Sciences, Vol. 19, No. 4, 2007, pp. 113-115. 